\title{
Automatic Segmentation on Glioblastoma Brain Tumor Magnetic Resonance Imaging Using Modified U-Net
}

\section{Hapsari Peni Agustin Tjahyaningtijas,3, Andi Kurniawan Nugroho1, Cucun Very Angkoso ${ }^{1}$, I Ketut Eddy Purnama1,2, Mauridhi Hery Purnomo ${ }^{1,2}$}

1Department of Electrical Engineering, Faculty of Intelligent Electrical and Informatics Technology, Institut Teknologi Sepuluh Nopember, Surabaya, Indonesia

${ }^{2}$ Department of Computer Engineering, Faculty of Intelligent Electrical and Informatics Technology, Institut Teknologi Sepuluh Nopember, Surabaya, Indonesia 3 Department of Electrical Engineering, Faculty of Engineering, Universitas Negeri

Surabaya, Surabaya, Indonesia

E-mail: hapsaripeni@unesa.ac.id,\{hapsari.17071, andinugroho.17071\}@mhs.its.ac.id, cucunvery@trunojoyo.ac.id, \{ketut,hery\}@ee.its.ac.id

Received March 9, 2020; Revised April 26, 2020; Accepted May 15, 2020

\begin{abstract}
Glioblastoma is listed as a malignant brain tumor. Due to its heterogeneous composition in one area of the tumor, the area of tumor is difficult to segment from healthy tissue. On the other side, the segmentation of brain tumor MRI imaging is also erroneous and takes time because of the large MRI image data. An automated segmentation approach based on fully convolutional architecture was developed to overcome the problem. One of fully convolutional network that used is U-Net framework. U-Net architecture is evaluated base on the number of epochs and drop-out values to achieve the most suitable architecture for the automatic segmentation of glioblastoma brain tumors. The segmentation performance evaluated using three models of U-Net architecture. The first architecture is the original U-Net, second is U-Net with all dropout in each convolutional layer and third is mU-Net that modified from both original U-Net and all drop-out U-Net. Through experimental findings, the most fitting architectural model is mU-Net architecture with an epoch number of 90 and a drop out layer value of 0.5 . The results of the segmentation performance are shown by a dice score value of 0.909 which is greater than that of the previous research.
\end{abstract}

Keywords: Brain tumor segmentation, fully convolutional network, U-Net, drop-out, dice score. 


\section{INTRODUCTION}

Tumor can be defined as the growth and division of cells in the body that are uncontrolled and unnatural [1]. The mass that grows from this unnatural growth and cell division in brain tissue is called a brain tumor. Brain tumor is one of the deadly cancers worldwide.

Based on the origin of tumor cell growth, brain tumors are classified into two types: primary tumors and metastatic brain tumors. Primary is the development of tumor cells arising from brain tissue itself and the metastatic growth from tumor cells originating from the spread of tumor cells from other regions of the body. Glioma is a type of brain tumor originating from glial cells. This type of tumor is a type of brain tumor that is the focus of current brain tumor segmentation research. The glioma is a general term that describes several types of brain tumors ranging from low levels such as astrocytomas and oligodendrogliomas to high levels, glioblastoma (grade IV). Glioblastoma is the most aggressive type of primary tumor [2]. In order to treat gliomas, medical experts perform several techniques including surgery, chemotherapy, and radiotherapy are techniques used to treat gliomas [3] Obviously, medical experts requires to observe the brain tumor images of the patient before applying any treatments.

Brain tumor imaging technology develops very rapidly and opens up the horizons of analytical thinking and study of brain anatomy and its functions. The recent imaging techniques such as X-ray computed tomography (CT) scans and Magnetic Resonance Imaging (MRI) is commonly used in brain tumors. Unlike CT scans, imaging of brain tumors using MRI gives a more detailed image while glioblastoma (GBM) tumors are depicted as images with diffuse areas, contrasts, and edema areas in the form of tentacles [4]. The advantage of using MRI include the acquisition of image data sets in large quantities with good quality [5]. In this regard, MRI modalities T1, T1-contrasted, T2 (spin-spin relaxation), and fluid attenuation inversion recovery (FLAIR) are needed for GBM imaging [4]. Meanwhile, the amount of MRI data set becomes an obstacle for clinical staff because the analysis process was done manually to get important information from an image. In general, manual analysis takes longer and causes errors in interpreting an image either between experts or inter experts so a computerized method is needed to improve the ability to diagnose GBM [6] The brain is composed of 3 types of tissue, namely white matter, gray matter, and cerebrospinal fluid. Tumor segmentation aims to determine the location of the tumor. The expansion of the tumor area called the active tumor tissue, necrotic, and edema. GBM is an infiltrative tumor, with very vague boundaries and difficult to distinguish from normal tissue.

In addition to manual segmentation, there are two methods of segmentation carried out in image segmentation, namely the semi-automatic and automatic segmentation methods. The semi-automatic segmentation method still requires human interaction when localizing the tumor area while the localization of the tumor area is completely done with the help of a 
computer in automatic segmentation. In the case of GBM segmentation, automatic segmentation was further evolved right now.

Most automated brain tumor segmentation utilizes hand-designed features [7]. These methods apply classic machine learning based on image segmented features. Such features become input with training procedures for the classifier which do not affect the character of the feature. These features become input for the classifier with training procedures that do not affect the nature of the feature. With an increasingly complex hierarchy of features, alternative approaches are needed to design representations of features that are adapted to the hierarchy [4]. Convolutional neural networks (CNN) have proved to be superior in feature hierarchy studies. In this study, we applied the CNN principle to analyze the hierarchy of features in segmenting GBM brain tumors automatically.

CNN application for segmentation is called Fully Convolutional Network (FCN), which distinguishes between CNN and FCN is that in FCN there is a convolutional layer after the last fully connected layer. The idea of adding a convolutional layer is to capture the global context of the scene. FCN does not use a "Dense" layer like CNN, but it uses a convolutional layer that acts as a dense layer [8]. FCN also requires CNN to transform pixel images into pixel categories [9]. In addition, FCN transforms height and weight of intermediate layer features back to the original reference image size through the transposed convolutional layer. There are several FCN for segmentation including FCN-32s, FCN-16s, FCN-8s, and U-net. In this study, U-Net was applied for automatic GBM brain tumor segmentation.

\section{RELATED WORKS}

Currently, an increasing number of studies in automatic brain tumor segmentation has performed. The approach in brain tumor segmentation is categorized into two groups. The first group focuses on generative model while other group concerns on a discriminative model [7][10].

The generative model has been established based on advanced information that distinguishes between tumor areas and healthy tissues. Determining the features of healthy tissues and tumors is a vital aspect during the research. Tumor tissue is known as a different shape from healthy tissues [11]. Typically, these approaches are based on an anatomical model produced after aligning 3D MRI in an atlas or a replica determined from multiple healthy brains. Throughout the form of the International Consortium for Brain Mapping (ICBM) brain atlas, this approach aligns the brain with the atlas and measures the posterior percentage of healthy tissue (white matter, gray matter, and cerebrospinal fluid). The tumor area is then found by localizing the area which is likely posterior below a certain threshold. A post-processing stage is then used to ensure sufficient spatial regularity [12]. Then the active contour method is initialized on this map and repeated until the posterior probability falls below a certain threshold. The active contour system [13] is one of the methods used for initialization. This 
method is highly reliant on alignment-based features and can encounter problems if the segmentation phase is carried out in large numbers and at the same time [14].

Another approach that is also used for segmenting brain tumors is the discriminative model. Unlike the generative model, this generative model uses prior knowledge in brain anatomy and works more on the extraction of low-level features in images. This model also provides relationships between features and labels as in the local histogram texture feature. The classic discriminative methods that are often used are Support Vector Machine (SVM) [10][15], decision forest[16], and random forest[17].

Other methods are known as Deep Learning deal with representation learning by automatically learning a hierarchy of increasingly complex features directly from data. These alternative approaches learn a hierarchy of complex features directly from domain data by using CNN that applied to medical image segmentation.

The use of CNN in data recognition and classification has begun to grow rapidly after the 2010 ImageNet Large Scale Visual Recognition Challenge competition. After some CNN architecture began to be known, such as Alexnet's eight-layer architecture [18], GoogLeNet has 22 layers [19], and the Resnet has more than 100 layers [20].

There are three layers in architecture CNN is based on its function, namely Convolutional Layer, Subsampling Layer, and Fully Connected Layer. CNN architecture usually consists of several attach the Convolutional Layer and Subsampling layers, followed by the Fully Connected layer. The convolutional layer is responsible for implementing features certain locations in all locations as a link layer that changes the input data feature map that has been convoluted with filters. The subsampling layer reduces the dimensions of the feature map by choosing pixel values based on certain rules to be output. The algorithm that is often used in the subsampling layer is a maxpooling operation. Fully connected layer function used to distinguish between classes and do nonlinear transformation to obtain the output value. Usually, the convolutional and subsampling layers are part of the features learning while fully connected layer is part of the classification.

The output of a convolutional layer will be input from the next convolutional layer. In the original condition, the output dimension is always smaller than the dimension input, which allows a lot of information to be lost. (except for using a 1x1 filter with stride 1). To overcome this, zero-padding (ZP) is used. ZP is a parameter that determines the number of pixels (containing the value 0 ) which is will be added to each side of the convolutional input layer. ZP layers as a manipulator so that the output dimension of the convolutional layer has the same size as the dimensions input. The addition of the ZP parameter causes a show CNN work can be improved, using convolutional layers is deeper so more and more features are can be extracted. This is what makes CNN increasingly widely used in the extraction and identification process of multidimensional data. 
Input image becomes the input of CNN used for segmentation by giving labels the pixels of the image. CNN performs the whole process repeatedly by paying attention to the features of each pixel until it has mapped the entire image.

Standard CNN has a layer fully connected, which can not accommodate the various input sizes [21] [22][23] . Fully convolutionary network (FCN) utilizes a convolution layer to process different input sizes and can perform faster. The final output layer has a wide receptive plane and corresponds to the height and width of the image, while the number of channels corresponds to the number of classes. The convolution layer classifies each pixel to decide the background of the image, including the position of the object.

FCN trained end-to-end, pixels-to-pixels on segmentation exceeds the state-of-the-art without further machinery. FCN train end-to-end includes pixel-wise prediction and supervised pretraining. Fully convolutional models of current networks estimate dense outputs from arbitrarily sized inputs. Both learning and inference are done by dense feed-forward computation and back-propagation. In the FCN network, upsampling layers allow pixelwise predictions and network learning with subsampled pooling. This approach is both asymptotically and fully accurate and precludes the need for problems in certain projects. Patchwise training is common [24][25], but lack the quality of fully convoluted training. One of the FCN networks is U-Net.

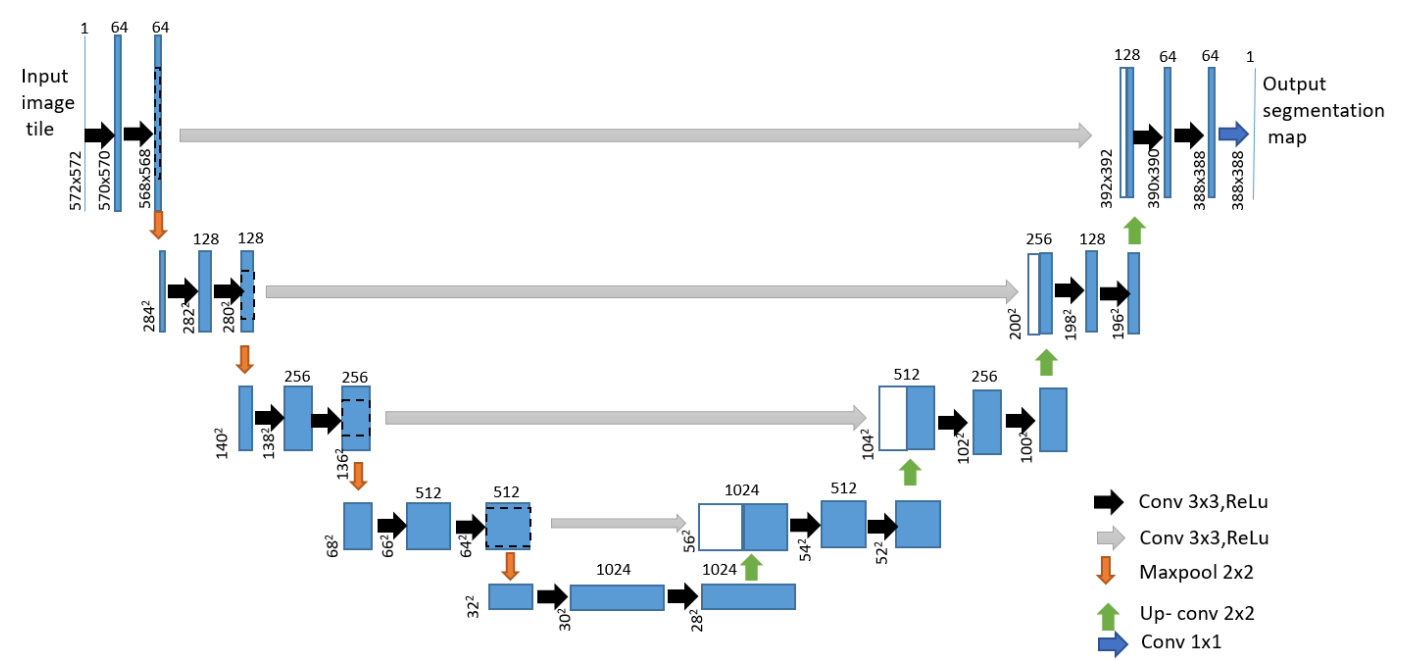

Figure 1. U-Net segmentation network

U-Net architecture consists of the contracting path and an expansive path (see Figure 1)[26]. The contracting path fits the CNN model, which consists of two convolution layers $3 \times 3$ ('unpadded convolution'). Each convolutional network uses the rectified linear unit (ReLU) activation function and the max-pooling layer $2 \times 2$ with stride 2 for downsampling. Every downsampling process, the number of features is doubled. The expansion path consists of two convolutional layers $3 \times 3$ with the ReLU activation function and the upsampling feature map $2 \times 2$ whose feature value 
is half the original. The link between the contracting path with the expansion path is concatenation. Concatenation will combine all the features of the contracting path to further experience the convolution process in the expansion path. The final layer in the expansion path consists of a $1 \times 1$ convolution layer which is used to map each vector feature component to the desired class. To enable smooth tiling of the output segmentation map in Figure 1, it is necessary to pick the input size in such a way that all $2 \times 2$ maxpooling operations are applied to the $\mathrm{x}$-and $\mathrm{y}$-size.

The input image and the segmentation results are used to train with the stochastic gradient descent. The segmented image generates an image with a constant boundary class which has a smaller size than the input image. A pixel-wise soft-max computes the energy function over the final feature map combined with the cross-entropy loss function. The cross-entropy each pixel position $x \in \Omega$, defined as:

$$
E=\sum_{x \in \Omega} w(x) \log \left(p_{l(x)}(x)\right)
$$

where $p_{l(x)}(x)$ is softmax function, $l: \Omega \rightarrow\{1, \ldots, \mathrm{K}\}$ is the true label of each pixel, and $\mathrm{w}: \Omega \rightarrow \mathrm{R}$ is weight map that gives some pixels more importance in the training.

The separation border is computed using morphological operations. The weight map is then computed as:

$$
w(x)=w_{c}(x)+w_{0} \cdot \exp \left(-\frac{(d 1(x)+d 2(x))^{2}}{2 \sigma^{2}}\right)
$$

where $w_{c}$ is the weight map to balance the class frequencies, $d 1$ denotes the distance to the border of the nearest cell, and $d 2$ denotes the distance to the border of the second nearest cell.

\section{ORIGINALITY}

CNN used a feature mapping process and accomplished it into a more nuanced feature. The application of CNN has been successfully applied to object recognition [18] [27]. For segmentation case, the step after change the feature map is followed by reconstructing the vector of the image. This is an enormous task because its vigorous to convert vector to an image and vice versa. Implementing U-Net will resolve the problem. U-Net uses the same feature map for a contraction to expand vectors to segmented images.

Inspired by the performance of the segmentation method in the related studies and the idea of efficient network segmentation, we adapted U-Net by adding a few modifications to its architecture to improve the performance of GBM segmentation. The U-Net adjustment is achieved by inserting and subtracting drop-out layer from the convolution layer in the original architecture. The first drop-out layer is applied to each convolutional layer, while the second drop-out layer is applied to just a few convolutional layers. In brief, this paper has the following contributions: 
1. We propose a fully automatic method with results currently ranked second on the BRATS 2018 scoreboard;

2. Analyze the various U-Net architectural designs to obtain the most appropriate U-Net GBM segmentation architecture according to the number of epoch and drop out layer.

This paper is presented in the following order. Part 1 is an introduction that explains the background of automatic GBM segmentation, part 2 presents related work presented in part 2, part 3 describes originality, part 4 explains about system design, part 5 contains experiments and data analysis, meanwhile, the conclusions are presented in section 6 .

\section{SYSTEM DESIGN}

The dataset used in this study is BraTS 2018. BraTS 2018 is a multimodal MRI scans with a ground truth label that has been manually revised by expert board-certified neuroradiologists. All imaging datasets were segmented manually by one to four raters, using the same annotation process, and their annotations were accepted by qualified neuroradiologists. In this experiment, figure 2 presents an overview of the proposed approach. There are three main stages: pre-processing, segmentation using U-Net, and post-processing.

\subsection{Pre-Processing}

The image obtained from the results of the MRI is highly influenced by the bias field. Bias field allows the intensity of the image to differ. The N4ITK approach is used to correct this [28]. The assumption for an MRI image consisting of several images is that each image has the same intensity distribution. This can not be done even if the N4ITK process has been used. The intensity of the image will vary considerably based on the time and position of the scan. The intensity normalization approach was used in each MRI picture to allow intensity uniform range. This intensity normalization method chooses a collection of MRI images to become a benchmark for other MRI images[29]. The chosen image must reflect the intensity of the percentile such that the value of the histogram is more uniform after the implementation of this method.

\subsection{U-Net Architecture}

In order to reduce overfitting, an ensemble of neural networks with different model configurations is present. However, it requires the additional computational expense of training and maintaining multiple models. The model can be used to simulate having a large number of different network architectures by randomly dropping out nodes during training. This is called dropout (DO). It offers a very low computational and remarkably effective regularization method. Therefore, this can reduce overfitting and improve generalization error in deep neural networks of all kinds. 
DO is a method used to reduce overfitting so that it is obtained more accurate classification results. Usually, overfitting occurs because the amount of training data is very large. During the training process, the DO parameter will negate the activation function randomly with certain probabilities. Whereas at the time testing, all activation functions will be used, but DO will provide weighting (scaling) with numbers certain. In many cases, the addition of DO will be effective to prevent overfitting because it reduces the correlation between neurons.

In this research, we discover the use of dropout regularization for reducing overfitting and improving the generalization of U-Net networks through the limitation of the data. We also analyze the performance of network by number of epoch.

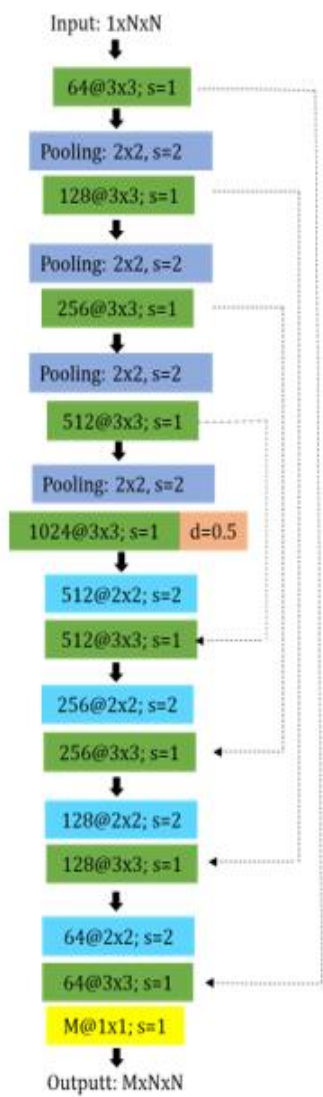

(a)

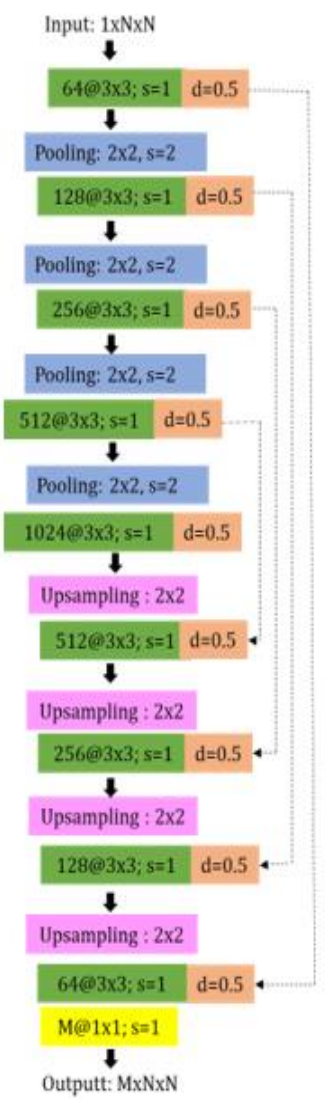

(b)

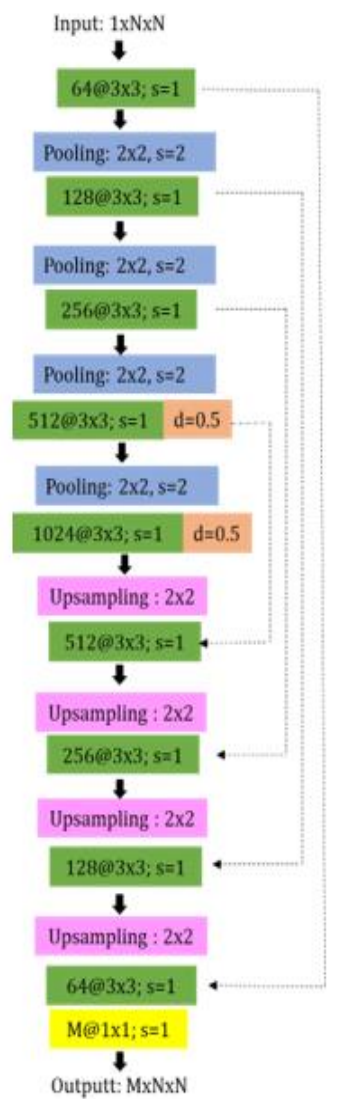

(c)

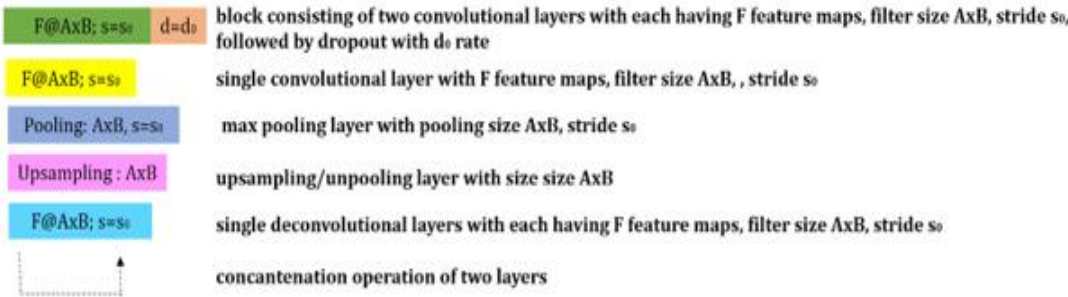

Figure 2. Cell layout in the system architecture for (a) original U-Net , (b) all dropout U-Net, and (c) modified U-Net (mU-Net) 
The U-Net architecture models (Figure 2) was used for segmentation, The networks are original U-Net architecture, all drop-out U-Net architecture by giving DO layers to each convolutional layer while mU-Net is the proposed modified U-Net architecture model by giving DO layers to a convolutional layer with 512 feature maps and a convolution layer with 1024 feature maps.

\subsection{Post-processing}

Quantitative evaluation of the model's performance on the test set is shown by the segmentation results. The tumor structures are grouped into 3 different tumor regions. This is mainly due to practical clinical applications. As described by Menze et al. [7], tumor regions are defined as whole tumor regions (including all four tumor structures), core tumor regions (including all tumor structures except "edema"), and enhancing tumor region (including the "enhanced tumor" structure). Our study evaluates only on complete tumor region.

The evaluation of the segmentations considered five metrics: Accuracy, Sensitivity, Specificity, Dice, and Jaccard similarity coefficients. The Dice measures the overlap between the manual and the automatic segmentation. The similarity coefficients accuracy, sensitivity, specificity, dice, and Jaccard might then be computed in the following way:

$$
\begin{aligned}
& \text { Accuracy }=\frac{T P+T N}{T P+F N+T N+F P} \\
& \text { Sensitifity }=\frac{T P}{T P-F N} \\
& \text { Specificity }=\frac{T P}{T P+F P} \\
& \text { Dice }=\frac{2 T P}{F P+2 T P-F N} \\
& \operatorname{Jaccard}\left(I, G_{I}\right)=\frac{2}{2-\text { Dice }}
\end{aligned}
$$

where TP, TN, FP, and FN are the numbers of true positive, true negative, false positive and false negative detections

\section{EXPERIMENT AND ANALYSIS}

The BRATS 2018 image dataset for GBM image was tested on various variations U-Net architecture as in Figure 2. The MRI data set image contain 102 patient of GBM. 85 data used for training and 17 data used for testing. The experiment results are presented in Figure 3 and Figure 4. A comparison of some U-Net parameters was done to get an optimal parameter. The parameters that need to be set on U-Net include different number of epochs or many iterations. Image batch size that determines many images used in one training process, many filters or convolution output map, stride size in the convolution process, kernel convolution size, and kernel pooling size. Comparisons were made using different parameter. However, some other 
parameters are given the same value for all U-Net architecture. Several parameters that compared including the epoch, the influence of DO, and classification accuracy. Whereas the other parameters were set with a default value i.e. size of stride $1 \times 1$, kernel $3 \times 3$, pooling kernel $2 \times 2$, and using Adam's optimization function as an optimizer. The image used as input from $\mathrm{U}$-Net is a grayscale image with $256 \times 256$ pixels.

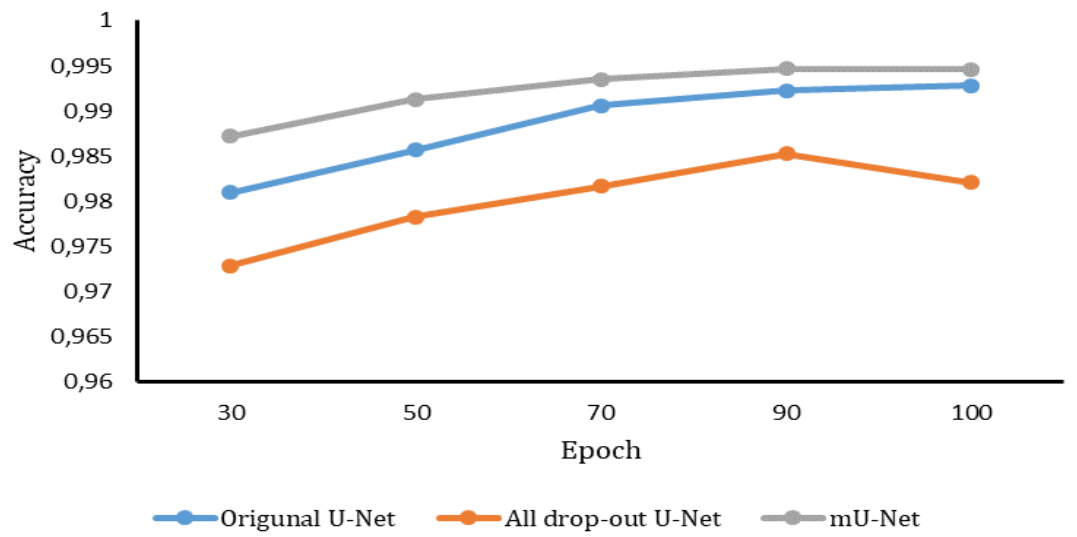

Figure 3. Level of accuracy with various epoch

The first experiment was made by making constant batch values at 32 with various epochs. The results of the experiment are shown in Figure 3. It appears that the greater the epoch, the higher the accuracy is obtained.

The highest accuracy, of $99.47 \%$, is given by the mU-Net architecture with 100 number of epoch. For the overall U-Net architecture that was approved, all drop-out U-Net provides the lowest performance when performing GBM image segmentation, which results in an accuracy of 98.51.\% with 30 epoch. Therefore, it can be concluded that the highest accuracy is obtained in the GBM image dataset with the number of epochs 90 and 100. There is a general trend for all architectural designs that, after the maximum accuracy achieved, the accuracy value will stagnate even though the number of epochs is continuously increased.

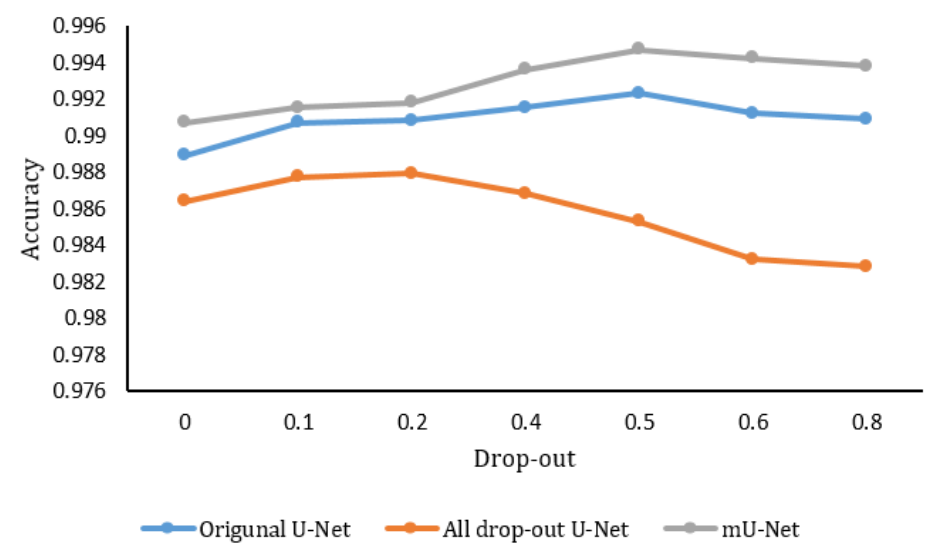

Figure 4. Level of accuracy result with various Drop-out number 
The other experiment was adding a DO layer to the feature extraction process to determine the level of significance in influencing system accuracy. The DO layer is inserted after the convolutional layer to eliminating overfitting. The epoch was set at 90 and batch at 32, and other parameters were the same for the size of stride. DO values vary in the range of $0-0.8$. The results were presented in Figure 4.

From Figure 4, it can be seen that the addition of DO layers for a certain DO value has an impact on increasing accuracy in all three U-Net architecture that had been tested. Increasing accuracy was evaluated for DO value from 0 to 0.8 for all architectures. In this range, the best performance is carried out by the mU-Net architecture for the GBM image dataset, the accuracy increases from 99\% to $99.47 \%$ (Increased 0.47\%). Whereas all drop-out UNet with the GBM dataset fixes an increase in the range of $98.64 \%-98.74 \%$ is approved given the DO layer from 0 to 0.04 .

For DO value of 0.5 , all U-Net architectural provide maximum accuracy. mU-Net provides the highest accuracy of $99.47 \%$ in the GBM segmentation. In line with increasing DO value will decrease the accuracy level of U-Net. The optimal DO value in the GBM image segmentation proceeds by 0.5 . It is shown that the accuracy of the system follows the parabolic curve with a maximum value of 0.5 .

Figure 5 displays the segmentation findings for the whole tumor region utilizing three U-Net segmentation architecture. The figure indicates that $\mathrm{mU}$ Net provides greater results in segmentation than other approaches.

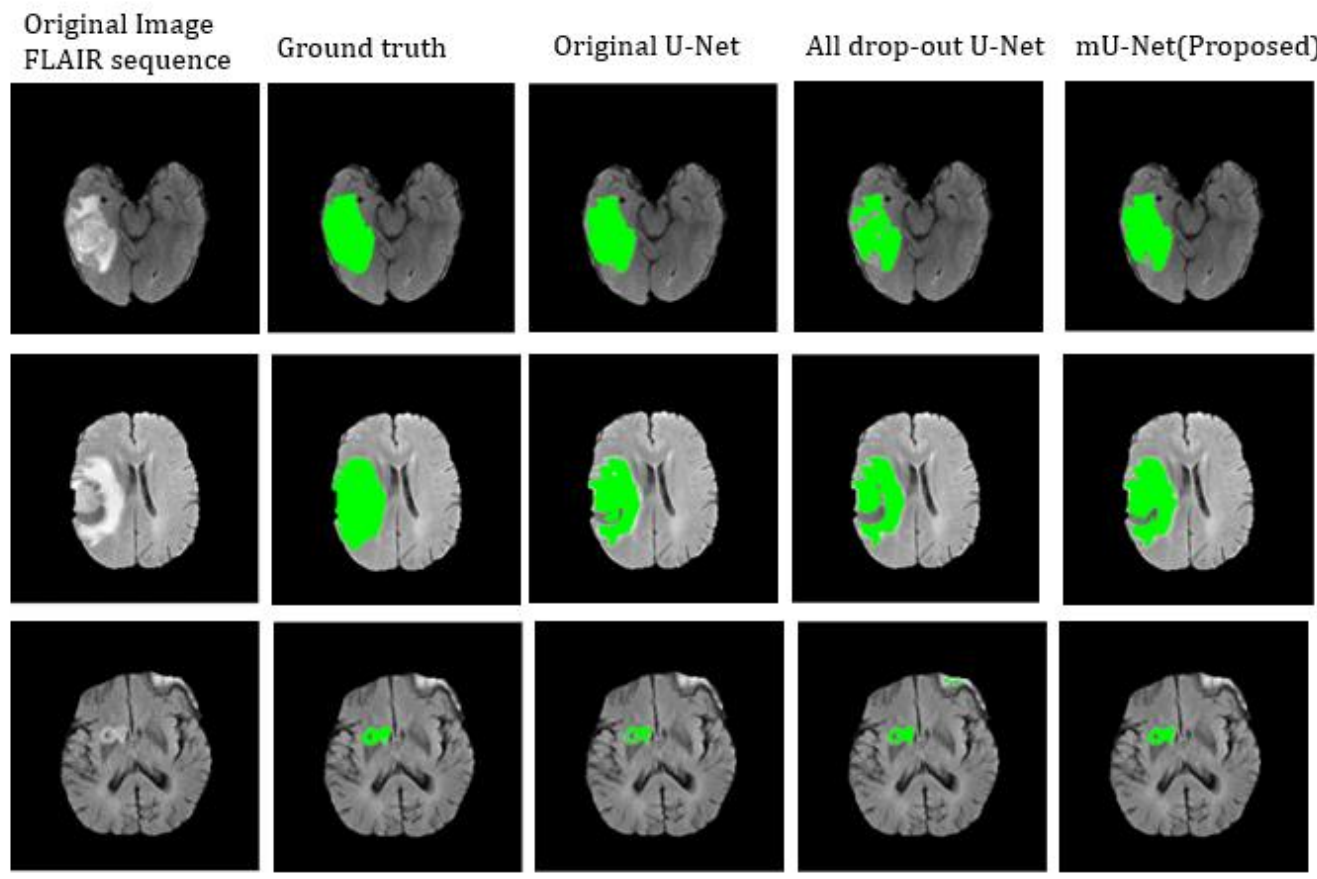

Figure 5. Example of GBM segmentation for whole tumor area, showing the effect of the component of proposed method. 
From the image segmentation result, mU-Net appears to be able to segment the GBM tumor region excellently, even in narrow tumor areas. In very heterogeneous and diverse tumor regions where the GBM region comprises of multiple tumor compartments, mU-net may even segment very well, although the other two segmentation methods also encounter limitations in the segmentation of the two regions. Defined from Table 1, the performance of segmentation output utilizing mU-Net is higher than the original U-Net and all drop-out U-Net with the accuracy of 0.995 , a sensitivity of 0.969 , a specificity of 0.995 , a dice score of 0.909 , and a Jaccard score of 0.834 .

Table 1. Segmentation comparison performance of U-Net for the whole GBM tumor.

\begin{tabular}{|c|c|c|c|}
\hline Performance & $\begin{array}{c}\text { Original } \\
\text { U-Net }\end{array}$ & $\begin{array}{c}\text { All drop-out } \\
\text { U-Net2 }\end{array}$ & $\begin{array}{c}\text { mU-Net3 } \\
\text { (proposed) }\end{array}$ \\
\hline Accuracy & 0.993 & 0.992 & 0.995 \\
\hline Sensitifity & 0.976 & 0.963 & 0.969 \\
\hline Specifity & 0.993 & 0.992 & 0.995 \\
\hline Dice Score & 0.871 & 0.858 & 0.909 \\
\hline Jaccard & 0.775 & 0.754 & 0.834 \\
\hline
\end{tabular}

The effect of the DO layer on the U-Net can be shown by contrasting the network with and without the DO layer. Throughout the original U-Net network, the D0 layer is a $1024 @ 3 \times 3$ convolutional layer, compared to the U-Net network by applying a DO layer on each convolutionary layer. The results obtained indicate that the addition of DO layers to each convolutional network can not improve the accuracy of the segmentation. The modification was made by applying the DO layer to not all convolutional layers. The maximum accuracy value was improved 0.002 when the DO layer was put on a $512 @ 3 x 3$ convolutional layer (Table 1). This result shows that applying DO layers to all convolutional layers on the U-Net will not automatically improve the accuracy of the segmentation, however, we need to be vigilant when applying them to the network. It is worth remembering that deciding which layers to train with dropout is an open problem, as several considerations (such as network architecture and even training data) have a major effect on performance. The right dropout regularization approach may be very specific for various network architectures.

The segmentation result of mU-Net is contrasted with the previous method, the findings of the new approach have a better meaning with a discrepancy in the dice score of 0.001 (Table 2). 
Table 2. Dice of our method on a validation set of BraTS data set

\begin{tabular}{|l|c|c|c|c|}
\hline \multicolumn{1}{|c|}{ Networks } & Data & $\begin{array}{c}\text { Level of user } \\
\text { interaction }\end{array}$ & Grade & Dice Score \\
\hline $\begin{array}{l}\text { Human } \\
\text { rater[7] }\end{array}$ & BraTS 2015 & Manual & Combined & 0.88 \\
\hline $\begin{array}{l}\text { CNN with small } \\
\text { 3x3 kernel [30] }\end{array}$ & BraTS 2015 & Fully automatic & GBM & 0.88 \\
\hline $\begin{array}{l}\text { Input Cascade } \\
\text { CNN [4] }\end{array}$ & BraTS 2015 & Fully automatic & Combined & 0.79 \\
\hline $\begin{array}{l}\text { DeepMedic } \\
\text { [31] }\end{array}$ & BraTS 2016 & Fully automatic & Combined & 0.9 \\
\hline $\begin{array}{l}\text { No new- } \\
\text { Net[32] }\end{array}$ & BraTS 2017 & Fully automatic & Combined & 0.896 \\
\hline $\begin{array}{l}\text { Cascaded CNN } \\
\text { [33] }\end{array}$ & BraTS 2018 & Fully automatic & Combined & 0.908 \\
\hline $\begin{array}{l}\text { mU-Net } \\
\text { (proposed) }\end{array}$ & BraTS 2018 & Fully automatic & GBM & 0.909 \\
\hline
\end{tabular}

CNN model with small size filter $3 \times 3$ had been done for automatic segmentation[30]. This approach was used to add convolutional layers without decreasing the performance of the filter. The proposed CNN has 11 layers of depth consisting of 6 convolutional layers followed by 3 fullyconnected layers with 2 max-pooling using the BRATS data set with a dice value of 0.88. Cascade CNN [4] has also been used in automatic brain tumor segmentation. The architecture consists of two CNN paths, each consisting of 2 convolutional layers, max-out, and max pooling. This architecture could segment brain tumors with a dice coefficient value of 0.79 . The architecture of mU-Net consists of 9 convolutional layers with DO layer at fourth and fifth convolutional layer, 4 max-pooling, and 4 upsampling layers. The proposed network used upsampling layer to map the feature to reconstruct the image.

Based on the results of comparisons in Table 2, the automatic segmentation method using mU-Net can be used in automatic segmentation of GBM brain tumors. mU-Net uses DO layers not on all convolutional layers compared with original U-Net and all drop-out U-Net. It indicates that the addition of the DO layer to the convolutional layer does not automatically boost segmentation performance. Adding DO layer is necessary to consider the balancing of data between training, validation, and testing. For unbalanced data, the addition of DO layers can improve segmentation performance by reducing overfitting.

\section{CONCLUSION}

This paper proposed an end-to-end approach for automatic GBM brain tumor segmentation images. We introduced and evaluated three U-Net architectures that achieved high overlap test scores for whole tumor GBM area on the BraTS'18 public dataset. The performance score is 0.995 for accuracy, a sensitivity of 0.969 , a specificity of 0.995 , a dice score of 0.909 , 
and 0.834 for a Jaccard score. Overall results show that adding more regularization by adding drop out on specific layers can improve the segmentation of objects.

For further research development, it is necessary to segment GBM brain tumor not only in the whole tumor area, but also in other tumor area segments such as enhancing tumor, edema, and necrosis for all MRI modalities.

\section{Acknowledgments}

This study was supported through Indonesian education scholarship program (BPPDN) by Indonesian Government.

\section{REFERENCES}

[1] L. M. DeAngelis, Medical progress: Brain tumors, N. Engl. J. Med., vol. 344, no. 2, pp. 114-123, 2001.

[2] T. Jiang, Y. Mao, W. Ma, Q. Mao, Y. You, X. Yang, C. Jiang, C. Kang, X. Li, L. Chen, X. Qiu, W. Wang, W. Li, Y. Yao, S. Li, S. Li, A. Wu, K. Sai, H. Bai, G. Li, B. Chen, K. Yao, X. Wei, X. Liu, Z. Zhang, Y. Dai, S. Lv, L. Wang, Z. Lin, J. Dong, G. Xu, X. Ma, J. Cai, W. Zhang, H. Wang, L. Chen, C. Zhang, P. Yang, W. Yan, Z. Liu, H. Hu, J. Chen, Y. Liu, Y. Yang, Z. Wang, Z. Wang, Y. Wang, G. You, L. Han, Z. Bao, Y. Liu, Y. Wang, X. Fan, S. Liu, X. Liu, Y. Wang, and Q. Wang, CGCG clinical practice guidelines for the management of adult diffuse gliomas, Cancer Lett., vol. 375, no. 2, pp. 263-273, Jun. 2016.

[3] R. Stupp, M. Brada, M. J. van den Bent, J. C. Tonn, and G. Pentheroudakis, High-Grade Malignant Glioma ESMO Clinical Practice Guidelines, Ann. Oncol., vol. 25, no. April, pp. 93-101, 2014.

[4] M. Havaei, A. Davy, D. Warde-Farley, A. Biard, A. Courville, Y. Bengio, C. Pal, P.-M. Jodoin, and H. Larochelle, Brain tumor segmentation with Deep Neural Networks, Med. Image Anal., vol. 35, pp. 18-31, Jan. 2017.

[5] G. Mohan and M. M. Subashini, MRI based medical image analysis: Survey on brain tumor grade classification, Biomed. Signal Process. Control, vol. 39, pp. 139-161, Jan. 2018.

[6] Jin Liu, Min Li, Jianxin Wang, Fangxiang Wu, Tianming Liu, and Yi Pan, A survey of MRI-based brain tumor segmentation methods, Tsinghua Sci. Technol., vol. 19, no. 6, pp. 578-595, Dec. 2014.

[7] B. H. Menze, A. Jakab, S. Bauer, J. Kalpathy-Cramer, K. Farahani, J. Kirby, Y. Burren, N. Porz, J. Slotboom, R. Wiest, L. Lanczi, E. Gerstner, M. A. Weber, T. Arbel, B. B. Avants, N. Ayache, P. Buendia, D. L. Collins, N. Cordier, J. J. Corso, A. Criminisi, T. Das, H. Delingette, Ç. Demiralp, C. R. Durst, M. Dojat, S. Doyle, J. Festa, F. Forbes, E. Geremia, B. Glocker, P. Golland, X. Guo, A. Hamamci, K. M. Iftekharuddin, R. Jena, N. M. John, E. 
Konukoglu, D. Lashkari, J. A. Mariz, R. Meier, S. Pereira, D. Precup, S. J. Price, T. R. Raviv, S. M. S. Reza, M. Ryan, D. Sarikaya, L. Schwartz, H. C. Shin, J. Shotton, C. A. Silva, N. Sousa, N. K. Subbanna, G. Szekely, T. J. Taylor, O. M. Thomas, N. J. Tustison, G. Unal, F. Vasseur, M. Wintermark, D. H. Ye, L. Zhao, B. Zhao, D. Zikic, M. Prastawa, M. Reyes, and K. Van Leemput, The Multimodal Brain Tumor Image Segmentation Benchmark (BRATS), IEEE Trans. Med. Imaging, vol. 34, no. 10, pp. 1993-2024, Oct. 2015.

[8] E. Shelhamer, J. Long, and T. Darrell, Fully Convolutional Networks for Semantic Segmentation, IEEE Trans. Pattern Anal. Mach. Intell., vol. 39, no. 4, pp. 640-651, Apr. 2017.

[9] J. Long, E. Shelhamer, and T. Darrell, Fully convolutional networks for semantic segmentation, in Proceedings of the IEEE Computer Society Conference on Computer Vision and Pattern Recognition, 2015, vol. 07-12-June, pp. 3431-3440.

[10] S. Bauer, R. Wiest, L.-P. Nolte, and M. Reyes, A survey of MRI-based medical image analysis for brain tumor studies, Phys. Med. Biol., vol. 58, no. 13, pp. R97-R129, Jul. 2013.

[11] L. P. Clarke, R. P. Velthuizen, M. Clark, J. Gaviria, L. Hall, D. Goldgof, R. Murtagh, S. Phuphanich, and S. Brem, MRI measurement of brain tumor response: comparison of visual metric and automatic segmentation., Magn. Reson. Imaging, vol. 16, no. 3, pp. 271-9, Apr. 1998.

[12] M. Prastawa, E. Bullitt, S. Ho, and G. Gerig, A brain tumor segmentation framework based on outlier detection, Med. Image Anal., vol. 8, no. 3, pp. 275-283, Sep. 2004.

[13] R. Maksimovic, S. Stankovic, and D. Milovanovic, Computed tomography image analyzer: 3D reconstruction and segmentation applying active contour models - 'snakes, Int. J. Med. Inform., vol. 58-59, pp. 29-37, Sep. 2000.

[14] N. Porz, S. Bauer, A. Pica, P. Schucht, J. Beck, R. K. Verma, J. Slotboom, M. Reyes, and R. Wiest, Multi-Modal Glioblastoma Segmentation: Man versus Machine, PLoS One, vol. 9, no. 5, p. e96873, May 2014.

[15] X. Bai and W. Wang, Saliency-SVM: An automatic approach for image segmentation, Neurocomputing, vol. 136, pp. 243-255, Jul. 2014.

[16] D. Zikic, B. Glocker, E. Konukoglu, A. Criminisi, C. Demiralp, J. Shotton, O. M. Thomas, T. Das, R. Jena, and S. J. Price, Decision Forests for Tissue-Specific Segmentation of High-Grade Gliomas in Multichannel MR, Springer, Berlin, Heidelberg, 2012, pp. 369-376.

[17] R. Jain, L. M. Poisson, D. Gutman, L. Scarpace, S. N. Hwang, C. A. Holder, M. Wintermark, A. Rao, R. R. Colen, J. Kirby, J. Freymann, C. C. Jaffe, T. Mikkelsen, and A. Flanders, Outcome prediction in patients with glioblastoma by using imaging, clinical, and genomic biomarkers: focus on the nonenhancing component of the tumor., Radiology, 
vol. 272, no. 2, pp. 484-93, Aug. 2014.

[18] A. Krizhevsky, I. Sutskever, and G. E. Hinton, ImageNet classification with deep convolutional neural networks, Commun. ACM, vol. 60, no. 6, pp. 84-90, Jun. 2017.

[19] C. Szegedy, W. Liu, Y. Jia, P. Sermanet, S. Reed, D. Anguelov, D. Erhan, V. Vanhoucke, and A. Rabinovich, Going deeper with convolutions, in Proceedings of the IEEE Computer Society Conference on Computer Vision and Pattern Recognition, 2015, vol. 07-12-June-2015, pp. 1-9.

[20] K. He, X. Zhang, S. Ren, and J. Sun, Deep residual learning for image recognition, in Proceedings of the IEEE Computer Society Conference on Computer Vision and Pattern Recognition, 2016, vol. 2016-December, pp. 770-778.

[21] S. Saxena and J. Verbeek, Convolutional Neural Fabrics, Adv. Neural Inf. Process. Syst., pp. 4060-4068, Jun. 2016.

[22] R. Rokhana, J. Priambodo, T. Karlita, I. M. G. Sunarya, E. M. Yuniarno, I. K. E. Purnama, and M. H. Purnomo, Convolutional Neural Network untuk Pendeteksian Patah Tulang Femur pada Citra Ultrasonik BMode, J. Nas. Tek. Elektro dan Teknol. Inf., vol. 8, no. 1, p. 59, Mar. 2019.

[23] A. Risnumawan, M. I. Perdana, Alif Habib Hidayatulloh, A. Khoirul Rizal, Indra Adji Sulistijono, Achmad Basuki, and Rokhmat Febrianto, Automatic Detection of Wrecked Airplanes from UAV Images, Emit. Int. J. Eng. Technol., vol. 7, no. 2, pp. 570-585, Dec. 2019.

[24] D. C. Cires, ancires,an, A. Giusti, L. M. Gambardella, and J. Urgen Schmidhuber, Deep Neural Networks Segment Neuronal Membranes in Electron Microscopy Images.

[25] C. Farabet, C. Couprie, L. Najman, and Y. Lecun, Learning hierarchical features for scene labeling, IEEE Trans. Pattern Anal. Mach. Intell., vol. 35, no. 8, pp. 1915-1929, 2013.

[26] O. Ronneberger, P. Fischer, and T. Brox, U-net: Convolutional networks for biomedical image segmentation, in Lecture Notes in Computer Science (including subseries Lecture Notes in Artificial Intelligence and Lecture Notes in Bioinformatics), 2015, vol. 9351, pp. 234-241.

[27] S. G, S. Kp, and V. R, Automated detection of diabetes using CNN and CNN-LSTM network and heart rate signals, Procedia Comput. Sci., vol. 132, pp. 1253-1262, Jan. 2018.

[28] N. J. Tustison, B. B. Avants, P. A. Cook, Y. Zheng, A. Egan, P. A. Yushkevich, and J. C. Gee, N4ITK: Improved N3 bias correction, IEEE Trans. Med. Imaging, vol. 29, no. 6, pp. 1310-1320, Jun. 2010.

[29] L. G. Nyúl, J. K. Udupa, and X. Zhang, New variants of a method of MRI scale standardization, IEEE Trans. Med. Imaging, vol. 19, no. 2, pp. 143-150, 2000.

[30] S. Pereira, A. Pinto, V. Alves, and C. A. Silva, Brain Tumor Segmentation Using Convolutional Neural Networks in MRI Images, IEEE Trans. Med. Imaging, vol. 35, no. 5, pp. 1240-1251, May 
2016

[31] K. Kamnitsas, E. Ferrante, S. Parisot, C. Ledig, A. V. Nori, A. Criminisi, D. Rueckert, and B. Glocker, DeepMedic for brain tumor segmentation, in Lecture Notes in Computer Science (including subseries Lecture Notes in Artificial Intelligence and Lecture Notes in Bioinformatics), 2016, vol. 10154 LNCS, pp. 138-149.

[32] F. Isensee, P. Kickingereder, W. Wick, M. Bendszus, and K. H. MaierHein, No new-net, in Lecture Notes in Computer Science (including subseries Lecture Notes in Artificial Intelligence and Lecture Notes in Bioinformatics), 2019, vol. 11384 LNCS, pp. 234-244.

[33] G. Wang, W. Li, S. Ourselin, and T. Vercauteren, Automatic Brain Tumor Segmentation Based on Cascaded Convolutional Neural Networks With Uncertainty Estimation, Front. Comput. Neurosci., vol. 13, p. 56, Aug. 2019. 